\title{
Data-Driven Forecasts of Regional Demand for Infrastructure Services
}

\author{
Jun $\mathrm{Ma}^{\mathrm{a}}$ \\ Rohan Wickramasuriya ${ }^{a}$ \\ Pascal Perez ${ }^{\mathrm{a}}$ \\ Murad Safadi $^{\mathrm{a}}$
}

\begin{abstract}
The socio-economic development and liveability of a region are affected to a great extent by the region's infrastructure services. Data-driven forecasting the demands for infrastructure utilities (for example, electricity, water, and waste) of a region becomes a challenging issue in the situation of highly integrative infrastructure networks and restricted data sharing, which involves handling temporary and spatial infrastructure utility data simultaneously and modelling the correlations between different infrastructure utilities and their interactions with relevant socio-economic and environmental indicators. Data mining and complex fuzzy set techniques are used to implement this kind of analytical capability in SMART Infrastructure Dashboard (SID). The developed forecasting method and technique can be used by local governmental agencies, infrastructure service designers and providers, and local communities for better governance, planning and delivering of effective and efficient infrastructure service and facility. It can also provide support evidence for a region's long-term sustainable planning and development.
\end{abstract}

Key words: Forecast; Infrastructure Services; Data mining; Fuzzy sets.

\section{Introduction}

Providing efficient and effective infrastructure services to local communities of a region is the base of improving its residential liveability, attracting commercial and industrial investments, advancing multi-cultural perspectives, and maintaining long-term sustainable development. To achieve this goal, an appropriate forecasting of regional demand of infrastructure services is a critical step ${ }^{1-2}$, which needs to consider not only provision of various infrastructure utilities and facilities but also the correlations among these infrastructure services and other relevant socio-economic, demographic, cultural, as well as environmental factors ${ }^{3}$. All sectors related to regional infrastructure services have produced huge amount of data, developing an appropriate data-driven forecasting of infrastructure service demands can benefit the local governmental agencies, infrastructure services planners, designers, and providers, local residences, and infrastructure researchers for better administration, development, use and understanding interactions between human behaviours, climate changes, and public services.

\footnotetext{
${ }^{a}$ SMART Infrastructure Facility, University of Wollongong, Wollongong, NSW 2522, Australia.

*Corresponding author: Jun Ma jma@uow.edu.au

http://dx.doi.org/10.14453/isngi2013.proc.30
} 
In this work, we introduce the practice of developing and employing a data-driven forecasting model of regional infrastructure service demands in the SMART Infrastructure Dashboard (SID) project being developed by the SMART Infrastructure Facility, the University of Wollongong. SID tries to provide support for regional infrastructure development through three integrated platforms, namely an information platform, an analysis platform, and a decision making platform. The data-driven forecasting model is one of functionalities provided by SID.

\section{Data-Driven Forecasting Challenges}

Developing a data-driven forecasting model of regional infrastructure service demands will provide challenges from data processing, data modelling and data visualisation. Data is the key to conducting forecasting of regional infrastructure service demands. Relevant data covers but is not limited to infrastructure utilities and facilities (such as electricity, water, and gas supply, waste collection, transportation, telecommunication and internet/wireless services), local area demographic characteristics (such as dwelling structures, population densities, population compositions), local socio-economic indicators (for examples, industrial structures, recreation traditions), and environmental measures (for examples, precipitation, temperatures, vegetation, geological structure and hazards). During long-term infrastructure development and practices, public service agencies, private providers and other infrastructure service related entities have accumulated mountains of infrastructure service data; however, these data are held separately or owned by individual entities due to all kinds of restrictions, policies and agreements. Data sharing is difficult and even prohibited. Although it has become an urgent requirement in infrastructure service communities, developing a datadriven forecasting model is far behind the needs of practitioners and commuters. Moreover, a data-driven forecasting model also needs to the face challenges in data feature comprehension and data quality regulation. In practice, it is unlikely to use perfect data without missing values and uncertainties.

Modelling techniques and methods are also challenges faced in a data-driven forecasting model. Data modelling is the foundation of forecasting demands of regional infrastructure services. Data mining, statistics, and machine learning techniques and methods have been widely used in building forecast models ${ }^{4-6}$. Existing modelling techniques and methods may not suit infrastructure practices. For instance, these modelling techniques and methods particularly focus on structured data, such as number, text, image, video, audio; however, infrastructure services data has greater emphasis on unstructured data, such as geographic distribution, interaction between different services. Hence, new modelling techniques and methods need to be investigated combining existing techniques and methods with infrastructure services features.

Data visualisation is an easily and commonly ignored challenge in a data-driven forecasting model $^{7}$. Data visualisation is not new - a simple scatter chart of a data set can be seen as a simple data visualisation. Data visualisation is an intuitionistic and effective way to reveal the inner patterns and trends hidden in the data. Data of infrastructure services has particular spatial and temporal features. For example, infrastructure utility consumptions change from place to place and from season to season. It is hard to demonstrate a forecasting model without considering these features in infrastructure service area. Using data visualisation techniques in a forecasting model can provide an intuitionistic and dynamic view of the changes of infrastructure service related factors and their interactions. Dashboard is an appropriate tool to implement data visualisation for infrastructure services. Recently, 
single infrastructure service dashboards have been developed; however, no crossinfrastructure service dashboard has been reported. SID tries to provide such one.

Combining data processing, data modelling, and data visualisation techniques, SMART Infrastructure Facility is developing a data-driven forecasting model of regional demands of infrastructure services in SID.

\section{Data-Driven Forecasting (DDF) of Demands of Regional Infrastructure Services}

The DDF focuses on the Illawarra region, NSW, Australia, which is a coastal area, south of the Greater Sydney Metropolitan Area. Traditionally, the region covers five local government areas (LGAs) - Wollongong, Shellharbour, Shoalhaven, Kiama, and Wingecarribee. Due to the fact that the Australian Bureau of Statistics (ABS) adopted a new geographic classification standard in its 2011 and will use it in its following censuses, the region boundary used in this paper is following the ABS 2006 census but the data is aligned to the ABS 2011 geographic classification hierarchy for future extension.

The data collected for the DDF includes electricity consumptions, water consumptions, regional temperature and rainfall measures, regional demographic profiles (such as population, dwelling types and compositions, household and family incomes, household and family compositions), community travel surveys and statistics.

The collected data is also dispersed in different spatial and temporal scales, aggregation and disaggregation methods are used to regulate the data to a given spatial and temporal metric according to the application requirement. Generally, two main spatial hierarchies are used in the DDF (see Figure 1). The DDF adopts three main temporal scales, namely, 'yearly', 'seasonal (quarterly)', 'monthly'. These scales are based on the analysis of the common data collecting periods.

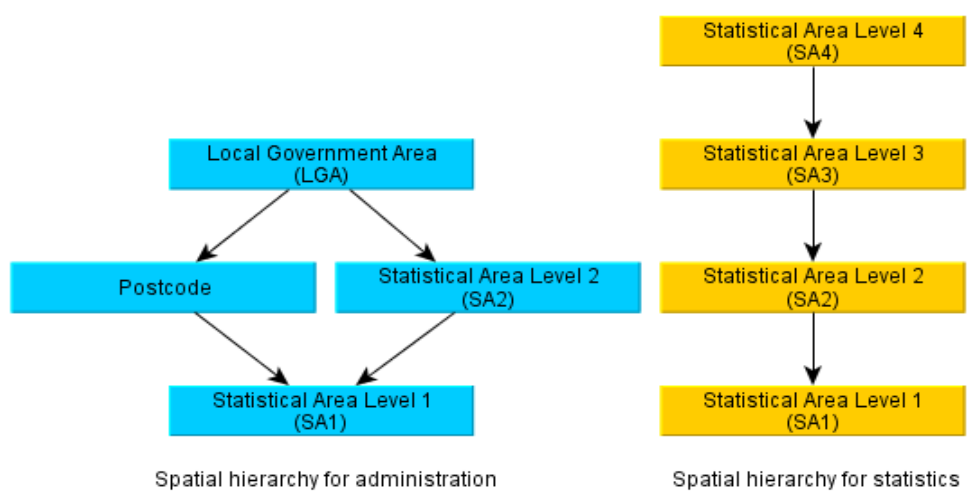

Figure 1. Spatial hierarchies for DDF.

The DDF adopts existing methods from data mining, machine learning, statistics and other relevant research areas. It also creates new methods according to the specific requirements and special features of infrastructure service data. For examples, fuzzy sets and complex fuzzy sets techniques have been used to describe the uncertainty and the seasonal features in infrastructure utility consumption; and classification and clustering techniques are used to 
extract correlations between different kind of infrastructure services and between infrastructure service, demography profiles and environmental indicators. These methods will be stored as stored in a repository for future revision and usage.

The outcomes of the DDF will be visualised through corresponding SID reports. Users thus can obtain the integrated and dynamic views of the interested information of demand of infrastructure services including its changes along a timeline and its trends in the future under the impacts of relevant factors.

\section{Case Study: Residential Electricity Consumption at Postcode Level}

This section describes the DDF through an example of forecasting residential electricity consumption. For simplification, we take 'postcode-seasonal' as the focused spatial-temporal scale.

Electricity is an important infrastructure utility. The demands of residential electricity have clear seasonal feature (as shown in Figure 2). At the same time, they are affected by residential characteristics and environment factors. In order to investigate the demands of residential electricity, we collected relevant data and conducted the DDF.

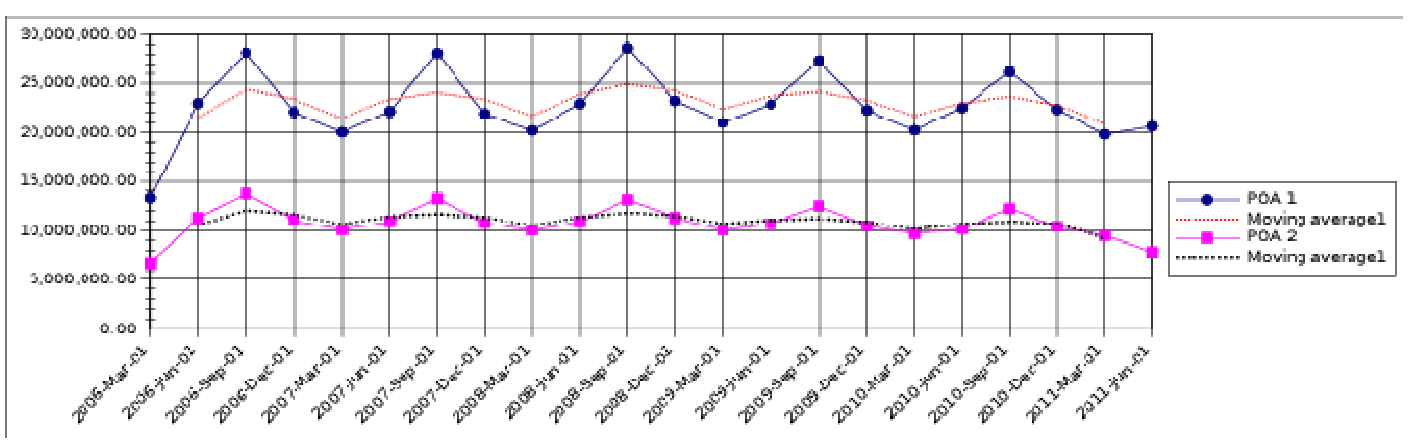

Figure 2: Comparison of residential electricity consumptions in two postcode areas (POAs).

The data collected for this study includes monthly rainfall and temperature records, population, ratios of different dwelling structures (separated house, semi-detached dwelling, flats or units), household median incomes, and seasonal electricity consumptions at census collection district (CCD) level (the smallest statistical unit in geographic classification in the ABS 2006 census) in five years from 2006 to 2011.

The obtained dataset is incomplete and contains many missing values and uncertainties. Main features of the data include:

- Rainfall and temperature data is collected from meteorological stations at specific locations.

- Population, dwelling statistics, household median incomes are only available for census years.

- Rainfall and electricity data are accumulated data which with time lags. 
Considering these features, some geospatial information processing techniques and interpolation methods are used to convert and transform the raw dataset to the given 'postcode-seasonal' scale. These techniques and methods include:

- Kirging technique is used to generate postcode level rainfall and temperature measures.

- Linear interpolation is used to generate seasonal population, dwelling statistics, household median incomes in non-census year.

- Fuzzy sets and complex fuzzy sets ${ }^{8-9}$ are used to describe the uncertainties in the rainfall and electricity consumption data.

After the necessary data pre-processing, a complete dataset is available for the DDF. Existing and developed software packages are applied to generate forecasting models. These models can be various forms such as neural networks, decision trees, logit models, as well as statistical models. Figure 3 shows two sections of decision tree models of residential electricity demands for the two postcode areas. The models are generated using the KNIME software.

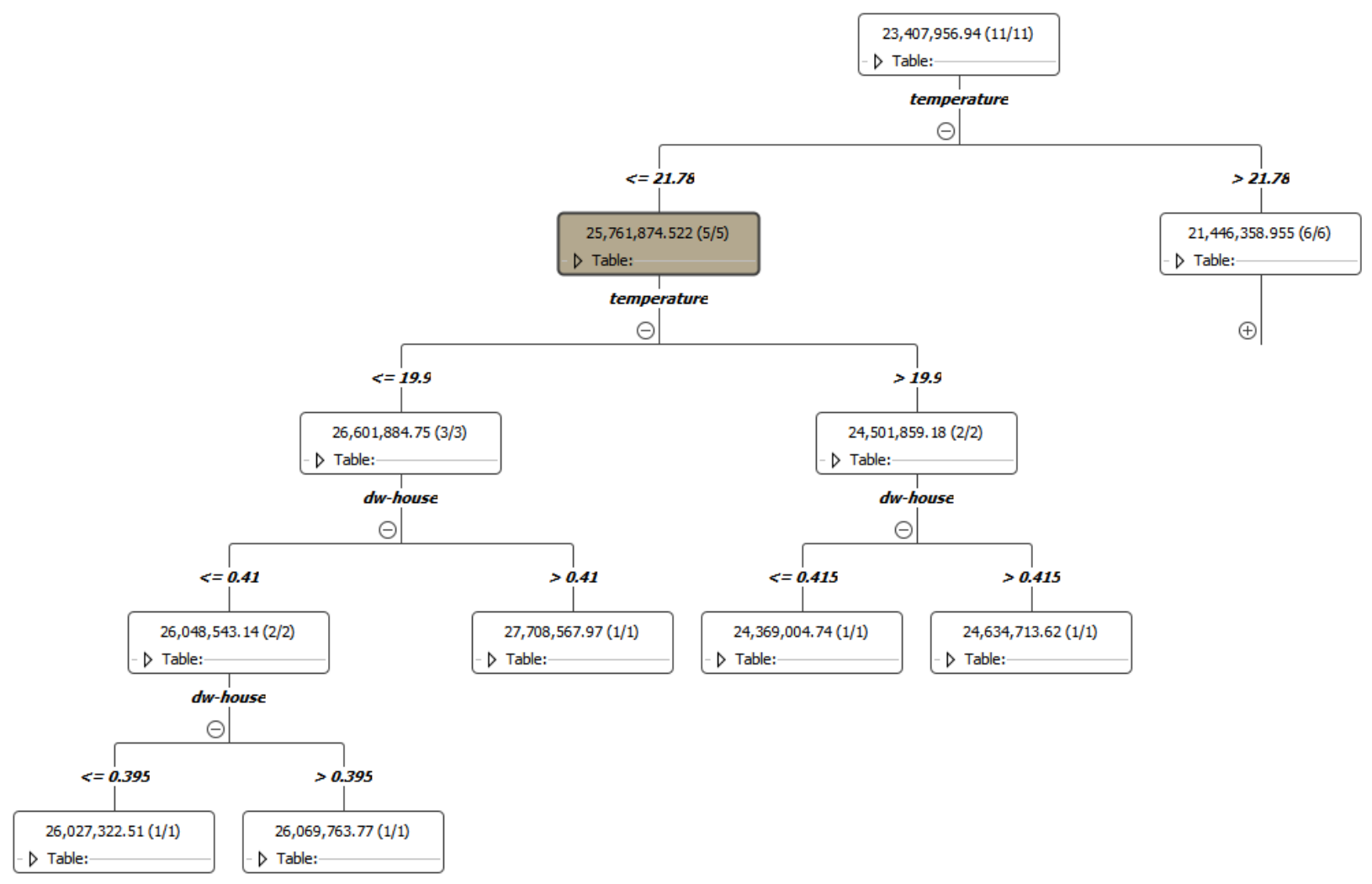

(a) Postcode area 1 


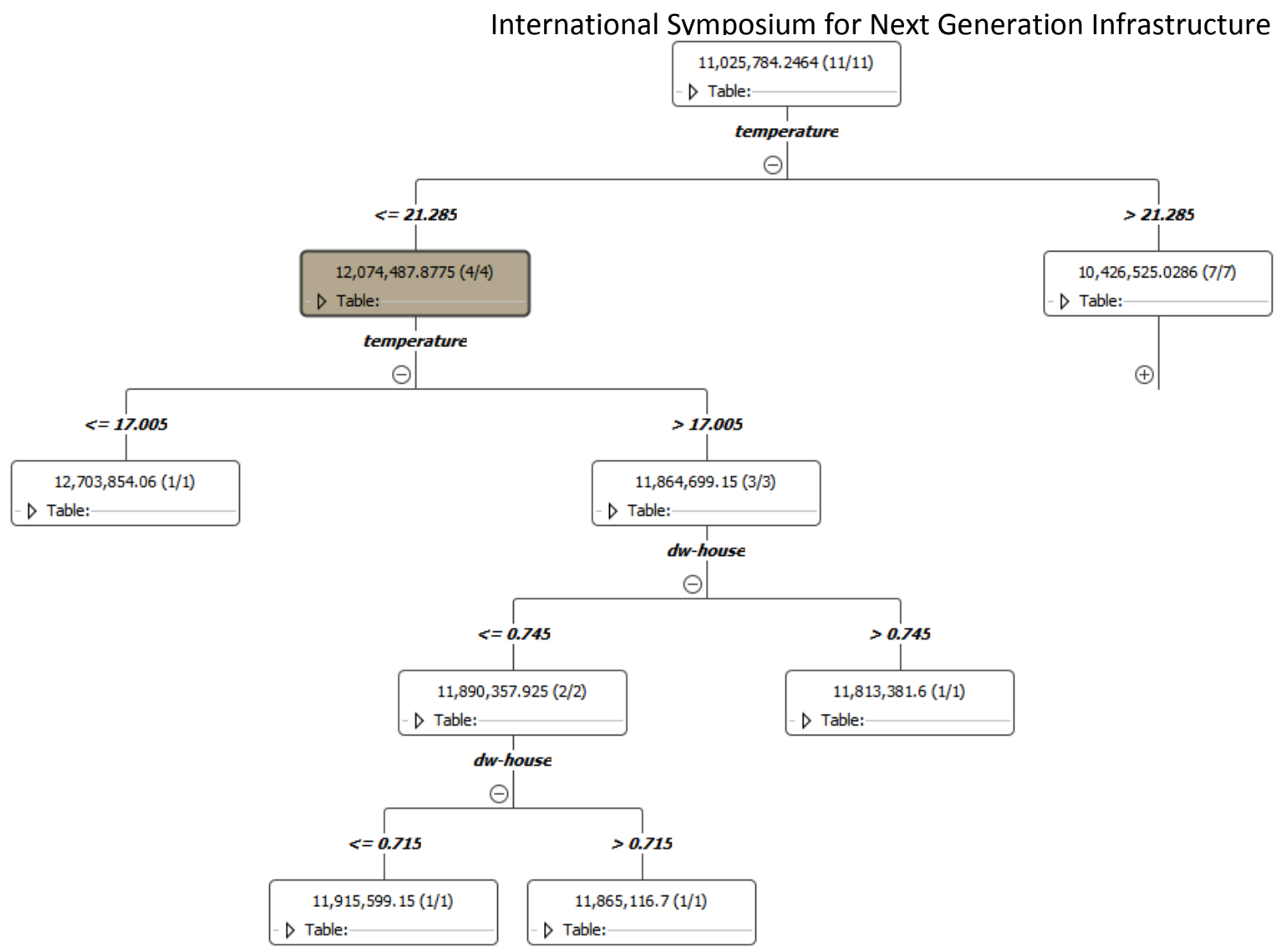

(b) Postcode area 2

Figure 3. Sections of decision tree forecasting models of residential electricity demands in two postcode areas.

\section{Conclusion and Future Work}

Forecasting the demands of regional infrastructure services has significant impact on better decision in infrastructure management, planning and designing. Data-driven forecasting is always an effective way to provide evidenced support for infrastructure development, particularly in today's big-data era. Provision of a data-driven forecast needs to overcome various barriers and challenges in data sharing and data modelling.

The SMART Infrastructure Facility is conducting relevant practice in its SID project which aims to provide regional infrastructure services information platform, analysis platform and decision platform. This paper briefly introduced the initial practice we have conducted in the project. To achieve the planned aims of the SID project, more works still need to be studied further such as forecasting techniques and methods, infrastructure services data understanding and processing.

\section{Acknowledgments}

Authors would like to acknowledge Sydney Water, Endeavour Energy, REMONDIS, Wollongong City Council, Shellharbour City Council, Kiama Municipal Council and Shoalhaven City Council. This research has been supported by the Australian National Data Service (ANDS) through their Application program. 


\section{References}

${ }^{1}$ Al-Ghandoor, A., Jaber, J., Al-Hinti, I., and Mansour, I., "Residential past and future energy consumption: potential savings and environmental impact", Renewable and Sustainable Energy Reviews, Vol. 13, 2009, pp. 1262-1274. http://dx.doi.org/10.1016/j.rser.2008.09.008

${ }^{2}$ Arghira, N., Hawarah, L., Ploix, S., and Jacomino, M., "Prediction of appliances energy use in smart homes", Energy, Vol. 48, 2012, pp. 128-134. http://dx.doi.org/10.1016/j.energy.2012.04.010

${ }^{3}$ Wang, S., Hong, L., and Chen, X., "Vulnerability analysis of interdependent infrastructure systems: A methodological framework", Physica A - Statistical Mechanics and Its Applications, Vol. 391, 2012, pp. 3323-3335.

${ }^{4}$ Chen, Z., Aghakhani, S., Man, J., and Dick, S., "ANCFIS: A neurofuzzy architecture employing complex fuzzy sets”, IEEE Transactions on Fuzzy Systems, Vol. 19, No. 2, 2011, pp. 305-322. http://dx.doi.org/10.1109/TFUZZ.2010.2096469

${ }^{5}$ Suganthi, L., and Samuel, A. A., "Energy models for demand forecasting - a review". Renewable and Sustainable Energy Reviews, Vol. 16, 2012, pp. 1223-1240. http://dx.doi.org/10.1016/j.rser.2011.08.014

${ }^{6}$ Swan, L. G., and Ugursal, V. I., "Modeling of end-use energy consumption in the residential sector: A review of modeling techniques", Renewable and Sustainable Energy Reviews, Vol. 13, 2009, pp.1819-1835. http://dx.doi.org/10.1016/j.rser.2008.09.033

${ }^{7}$ Heer, J., and Shneiderman, B., "Interactive dynamics for visual analysis", Communications of the ACM, Vol. 55, No. 4, 2012, pp. 45-54. http://dx.doi.org/10.1145/2133806.2133821

${ }^{8}$ Ramot, D., Milo, R., Friedman, M., and Kandel, A., "Complex fuzzy sets". IEEE Transactions on Fuzzy Systems, Vol. 10, No. 2, 2002, pp. 171-186. http://dx.doi.org/10.1109/91.995119

${ }^{9} \mathrm{Ma}$, J., Zhang, G., and Lu, J., "A method for multiple periodic factor prediction problems using complex fuzzy sets”, IEEE Transactions on Fuzzy Systems, Vol. 20, No. 1, 2012, pp. 32- 45. http://dx.doi.org/10.1109/TFUZZ.2011.2164084

${ }^{10}$ Escriva'-Escriva', G., A' lvarez Bel, C., Rolda'n-Blay, C., and Alc'azar-Ortega, M., "New artificial neural network prediction method for electrical consumption forecasting based on building end-uses", Energy and Buildings, Vol. 43, 2011, pp. 3112-3119. http://dx.doi.org/10.1016/j.enbuild.2011.08.008 